\title{
Cardiac Activation Associated with Non-Periodic Leg Movements in Comparison to Periodic Leg Movements during Sleep in Patients with Restless Legs Syndrome and Healthy Subjects
}

\author{
Min-Jong Kim ${ }^{1}$, Kwang Su Cha ${ }^{2}$, Tae-Joon Kim², Jin-Sun Jun ${ }^{3}$, Ki-Young Jung ${ }^{1,2,4,5}$ \\ ${ }^{1}$ Department of Neurology, Seoul National University College of Medicine, Seoul, \\ ${ }^{2}$ Department of Neurology, Seoul National University Hospital, Seoul, \\ ${ }^{3}$ Department of Neurology, School of Medicine, Kyungpook National University, Kyungpook National University Chilgok Hospital, Daegu, \\ ${ }^{4}$ Program in Neuroscience, Seoul National University College of Medicine, Seoul, \\ ${ }^{5}$ Neuroscience Research Institute, Seoul National University College of Medicine, Seoul, Korea
}

Received November 27, 2018 Revised December 24, 2018 Accepted December 28, 2018

Address for correspondence Ki-Young Jung, MD, PhD Department of Neurology, Seoul National University Hospital, Seoul National University College of Medicine, 101 Daehak-ro, Jongno-gu, Seoul 03080, Korea Tel: $+82-2-2072-4988$ Fax: +82-2-2072-2474 E-mail: jungky@snu.ac.kr
Objectives: Periodic leg movements in sleep (PLMS) are associated with arousals and autonomic activation, which may contribute to higher cardiovascular disease risk in patients with restless legs syndrome (RLS). Non-periodic leg movements in sleep (NPLM) are leg jerks in sleep that does not satisfy standard criteria of PLMS. The aim of this study was to evaluate impact of short-interval leg movements in sleep (SILMS) and isolated leg movements in sleep (ILMS) in comparison to PLMS on heart rate in both patients with RLS and healthy controls. Methods: Seven idiopathic RLS patients and 9 controls were enrolled in this study. Polysomnographic studies were analyzed and leg movements (LM) were automatically detected. NPLM can be classified as SILMS and ILMS. SILMS are LM separated by an inter-movement interval (IMI) shorter than $10 \mathrm{~s}$, and ILMS are LM with IMI longer than $90 \mathrm{~s}$. Frequency and heart rate associated with SILMS, ILMS, and PLMS in RLS patients were compared to those in controls. Heart rate change associated with LM were determined for a fixed time window. Results: Frequencies of SILMS and ILMS of patients with RLS were not significantly different to those of controls. RLS patients presented higher heart rate change associated with SILMS than PLMS before movement onset, while heart rate change associated with SILMS, ILMS, and PLMS were not different in the controls. Conclusions: Although the number of SILMS is not higher than PLMS, SILMS may have closely associated with higher cardiac activation of RLS than PLMS. Therefore, SILMS might be an important treatment target for patients with RLS to reduce long-term cardiovascular risk. Long-term prospective studies are needed to evaluate the relationship between NPLM and cardiovascular disease in patients with RLS.

J Sleep Med 2018;15(2):68-73 Restless legs syndrome.

\section{Introduction}

Periodic leg movements in sleep (PLMS) are repetitive leg jerks characterized by flexions at hip, knee, and ankle during sleep. PLMS are defined as leg movements (LM) lasting from $0.5 \mathrm{~s}$ to $10 \mathrm{~s}$, separated by an inter-movement interval (IMI) ranging between $10 \mathrm{~s}$ and $90 \mathrm{~s}$, and organized by at least four in a row according to World Association of Sleep Medicine (WASM) criteria. ${ }^{1}$ PLMS are present in more than $80 \%$ of pa-

This is an Open Access article distributed under the terms of the Creative Commons Attribution Non-Commercial License (https://creativecommons.org/licenses/by-nc/4.0) which permits unrestricted non-commercial use, distribution, and reproduction in any medium, provided the original work is properly cited. tients with restless legs syndrome (RLS) in North America, ${ }^{2}$ and in $60 \%$ of patients with RLS in Asia.,

PLMS are associated with electroencephalogram (EEG) arousals and sympathetic autonomic activations such as increase in heart rate and arterial blood pressure. It has been repeatedly reported that changes in EEG and heart rate start before the onset of PLMS. From this temporal relation, PLMS, EEG arousals and autonomic activations seem to be modulated by a complex dynamically interacting system of cortical and subcortical mechanisms. ${ }^{5}$ Repetitive nocturnal autonomic activations associated with PLMS might play a role in a higher cardiovascular risk in the patients with RLS. ${ }^{6}$ In addition, 
the presence of cerebral hemodynamics disturbances in RLS patients is associated with PLMS, which may contribute to an increased risk of cerebrovascular events described by epidemiologic studies. ${ }^{7,8}$

Non-periodic leg movements in sleep (NPLM) are leg jerks in sleep that does not satisfy WASM standards for PLMS. NPLM can be classified as short-interval leg movements in sleep (SILMS) and isolated leg movements in sleep (ILMS). SILMS are LM separated by an IMI shorter than $10 \mathrm{~s}$, and ILMS are LM with IMI longer than $90 \mathrm{~s}$.

Cardiac activation associated with NPLM in patients with RLS was analyzed by small number of studies. SILMS during non-rapid eye movement (NREM) sleep that were associated with arousal and a sequence of two LM (doublet) had a similar peak cardiac activation as that associated with PLMS, but had a significantly longer duration. ${ }^{9}$ Cardiac activation related to ILMS compared to that related to PLMS had conflicting results. In the study by Ferri et al., heart rate elevations seen with ILMS are higher than those seen with PLMS, ${ }^{5}$ while heart rate elevations with PLMS were higher than those with ILMS in the study by Guggisberg et al. ${ }^{10}$ Therefore, understanding the relationships between NPLM and heart rate and EEG arousal has important implications for evaluating the risks associated with cardiovascular disease in patients with RLS.

Previous studies of cardiac activation accompanied by NPLM were limited by number and conditions of LM analyzed. In addition, information on cardiac activation associated with NPLM in healthy subjects is not available. Further investigation is required to clarify impact of NPLM on heart rate in RLS patients during entire sleep stages.

The objective of this study was to evaluate impact of SILMS and ILMS in comparison to PLMS on heart rate in both patients with RLS and healthy controls. Subsequently, to investigate if there was RLS specific change in heart rate, we compared heart rate response seen in RLS with those seen in healthy controls.

\section{Methods}

\section{Subjects}

Patients were diagnosed with idiopathic RLS based on International Restless Legs Syndrome Study Group diagnostic criteria utilizing the validated Korean version of the Johns Hopkins telephone diagnostic questionnaire in face-to-face interviews. The questionnaire includes questions that help to exclude conditions that may mimic RLS. ${ }^{11}$ Severity of RLS was measured with International Restless Legs Syndrome Study Group Rating Scale (IRLS) score.

Patients with RLS were included in the study when they meet the following criteria: 1) aged over 30 years, 2) no prior treatment for RLS. The exclusion criteria were as follows: 1) apnea/hypopnea index $\geq 5,2$ ) comorbidities likely to be associated with secondary RLS (e.g., pregnancy, chronic kidney disease, or peripheral neuropathy). In addition, since the purpose of the study was to compare the cardiac impact of NPLM and PLMS, 3) patients without PLMS were also excluded. All patients underwent a neurological examination and routine blood tests (including serum iron, transferrin, ferritin, and blood urea nitrogen).

An age-matched group of healthy female subjects was recruited and served as controls. Control subjects were excluded if they were fulfilled at least one of followings; 1) apnea hypopnea index (AHI) $>5$, 2) periodic leg movements index (PLMI) $>15$, and 3) no PLMS.

All subjects conducted the Pittsburgh Sleep Quality Index (PSQI). Subjective sleep quality was measured with PSQI total score. Participants in the control group had no long-term use of medications to treat a psychiatric or neurological disorder or chronic disease and no history of sleep disorders as assessed using a sleep questionnaire. This study was approved by the Institutional Review Board (IRB) of Seoul National University Hospital (IRB no. 1705-118-855).

\section{Polysomnography and sleep scoring}

All subjects underwent a single overnight polysomnography (PSG) recording. The following signals were recorded: 19 EEG channels (10-20 system), chin electromyography (EMG), bilateral anterior tibial EMG, electrooculogram, and electrocardiogram (ECG). EEG signals were sampled at $200 \mathrm{~Hz}$. Sleep stage was scored in 30-s epochs according to the standard criteria described by American Academy of Sleep Medicine (AASM) manual for scoring sleep stage. ${ }^{12}$

Objective sleep quality was measured with polysomnographic findings including total sleep time (TST), sleep latency, wake time after sleep onset, sleep efficiency, NREM and rapid eye movement (REM) sleep \%, and arousal index.

\section{Analysis of leg movements}

LM were detected automatically during NREM and REM stage using Stanford EEG Viewer (SEV), which is a Matlab R2014 (The Mathworks, Natick, MA, USA) toolbox for viewing biological data collected from nocturnal PSG. ${ }^{13}$ All of the functions for leg movement detection in SEV were reviewed and modified appropriately in order to meet the latest WASM standards. Leg EMG signals were band-pass filtered at 10-99 $\mathrm{Hz}$ and rectified. LM were automatically detected subsequently using SEV functions. LM during wake stage were excluded. Respiratory event-related LM were also excluded in accordance with WASM standards. ${ }^{14}$ Extracted individual LMs were confirmed by visual inspection. 
IMI were defined as time difference between onset of later LM and onset of earlier LM. Distribution of IMI was analyzed and plotted as a histogram for each group. LM were classified into 3 groups according to previously described definitions: PLMS were LM with 1) duration of 0.5-10 s, 2) IMI ranging between $10 \mathrm{~s}$ and $90 \mathrm{~s}, 3) 4$ or more LM in a row. SILMS were LM with IMI shorter than $10 \mathrm{~s}$ and ILMS were LM with IMI longer than $90 \mathrm{~s}$. Frequencies of LM are assessed with index, which is the number of LM per sleep hour (Supplementary Table 1 in the online-only Data Supplement).

\section{Analysis of heart rate change}

Heart rate was calculated from R-R intervals detected from ECG and interpolated linearly at $100 \mathrm{~Hz}$. In order to reduce baseline variability, a fixed time window of $50 \mathrm{~s}$ (20 s preceding and $30 \mathrm{~s}$ following each LM onset) was used. Change of heart rate was expressed as a percentage of the baseline heart rate, which was average heart rate for first $10 \mathrm{~s}$ of each time window. Normalized heart rate change in a time window of $50 \mathrm{~s}$ around each LM was averaged for each individual and then for the group subsequently for 3 LM types.

\section{Statistical analysis}

Because of limited numbers of subjects enrolled in the study, nonparametric statistical analysis was applied. Comparisons were performed by means of Mann-Whitney U-test for independent data sets, Wilcoxon signed rank test for paired samples or Kruskal-Wallis test for three data sets. Difference with $p<0.05$ was considered significant. In comparison of heart rate change by LM types at each time point, $p<0.005$ was adopted as a significant level for considering multiple testing problem.

\section{Results}

\section{Demographic and clinical data}

Seventeen drug-naïve RLS patients and 16 age-matched healthy controls initially enrolled in this study. Ten patients were excluded from the study due to following reasons; 1) six patients had $\mathrm{AHI}>5,2$ ) one patient was younger than 30 years, and 3) no PLMS in one patient. Three control subjects were excluded because of AHI $>5(n=1)$, PLMI $(>15: n=1$, and no PLMS: $n=1)$. Four control subjects were further excluded owing to poor data quality in either PLMS or ECG (Fig. 1)

Seven drug-naïve patients with idiopathic RLS (6 females, median age: 49.00 years, interquartile range: $40.00-53.00)$ and nine age-matched healthy female controls were enrolled in the study (median age: 51.00 years, interquartile range: 41.50-54.50).

Table 1 reports the demographic, clinical data of each group

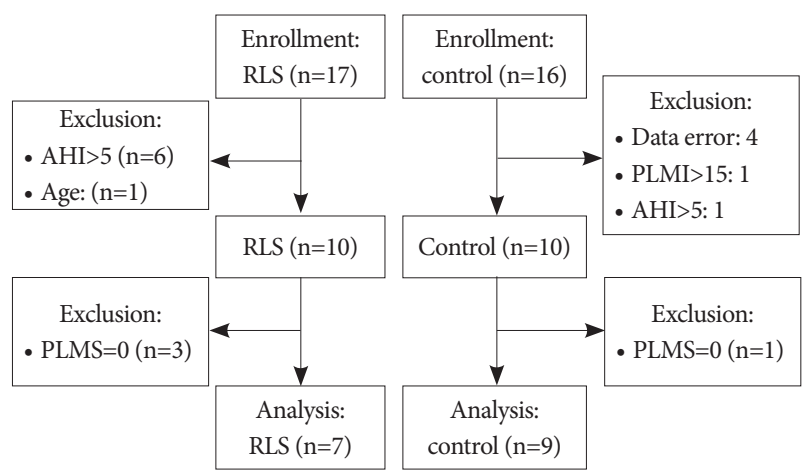

Figure 1. Flow chart of participant exclusions and inclusions. RLS: restless legs syndrome, AHI: apnea hypopnea index, PLMI: periodic leg movements Index, PLMS: periodic leg movements in sleep.

and comparison between groups. Patients with RLS have significantly higher PSQI and shorter TST compared to healthy controls. This result indicates that patients with RLS have worse sleep quality than controls, both subjectively and objectively. Patients with RLS have median IRLS score of 27.00 (interquartile range: 22.00-35.00).

\section{Distribution of inter-movement intervals}

IMI of the RLS group shows bimodal distribution with 2 peaks at 0-2 s and 18-20 s, while IMI of the control group shows unimodal distribution with a single peak at 2-4 s (Fig. 2). The second peak in the patient group represents PLMS, which is absent in the control group.

\section{Frequencies of leg movements}

Patients have a significant higher PLMS index than controls. SILMS and ILMS indices in patients are not significantly different from those in controls (Table 2).

\section{Comparison of average heart rate change among three LM types}

In the RLS group, SILMS and ILMS presented higher heart rate change compare to PLMS ( $p<0.02 ; p<0.03)$. However, no significant differences of average heart rate change among three LM types were observed in the control group (Table 3).

\section{Time course of heart rate change accompanied with leg movements}

Heart rate begins to increase prior to the onset of LM by 5$10 \mathrm{~s}$ in both groups (Fig. 3), which is consistent with previous studies. ${ }^{5,9}$ In patients with RLS, heart rate change in relation to LM is significantly different by LM types. SILMS are accompanied with the greatest and the longest increase in heart rate change while PLMS are associated with the lowest and the shortest heart rate change. The difference between heart rate related to SILMS and PLMS is significant for 6-7 s as well 
Table 1. Clinical characteristics and polysomnographic findings of patients and controls

\begin{tabular}{lccc}
\hline & RLS $(\mathrm{n}=7)$ & Control $(\mathrm{n}=9)$ & $p$-value \\
\hline Clinical characteristics & & & 0.82 \\
Age (year) & $49.00(40.00-53.00)$ & $51.00(41.50-54.50)$ & 100.00 \\
Female sex (\%) & 85.71 & & $<0.01^{*}$ \\
Disease duration (year) & $12.00(8.00-22.00)$ & & \\
IRLS score & $27.00(22.00-35.00)$ & $5.00(3.00-6.50)$ & $<0.01^{*}$ \\
PSQI & $10.00(8.00-18.00)$ & & 0.70 \\
Polysomnographic findings & & $445.40(412.75-464.45)$ & 0.11 \\
TST (min) & $386.00(285.00-392.00)$ & $8.00(4.00-11.50)$ & 0.12 \\
Sleep latency (min) & $4.50(1.50-12.50)$ & $23.00(15.75-32.80)$ & 0.25 \\
WASO (min) & $49.90(22.30-169.40)$ & $94.10(91.75-94.65)$ & 0.55 \\
Sleep efficiency (\%) & $86.50(62.10-94.30)$ & $13.12(9.85-18.63)$ & 0.92 \\
N1 (\%) & $16.60(13.70-21.80)$ & $45.20(38.95-47.85)$ & 0.61 \\
N2 (\%) & $48.60(37.70-52.60)$ & $15.90(10.85-20.25)$ & 0.09 \\
N3 (\%) & $15.10(7.90-22.10)$ & $25.35(19.05-27.70)$ & $11.70(9.75-17.00)$ \\
REM stage (\%) & $19.20(17.10-28.70)$ & $23.00(18.20-25.10)$ & \\
Arousal index & & & \\
\hline
\end{tabular}

Data are presented as median (interquartile range). Mann-Whitney $U$ test. ${ }^{*} p$-values $<0.05$. RLS: restless legs syndrome, IRLS: International Restless Legs Syndrome Study Group Rating Scale, PSQI: Pittsburgh Sleep Quality Index, TST: total sleep time, WASO: wake time after sleep onset, REM: rapid eye movement
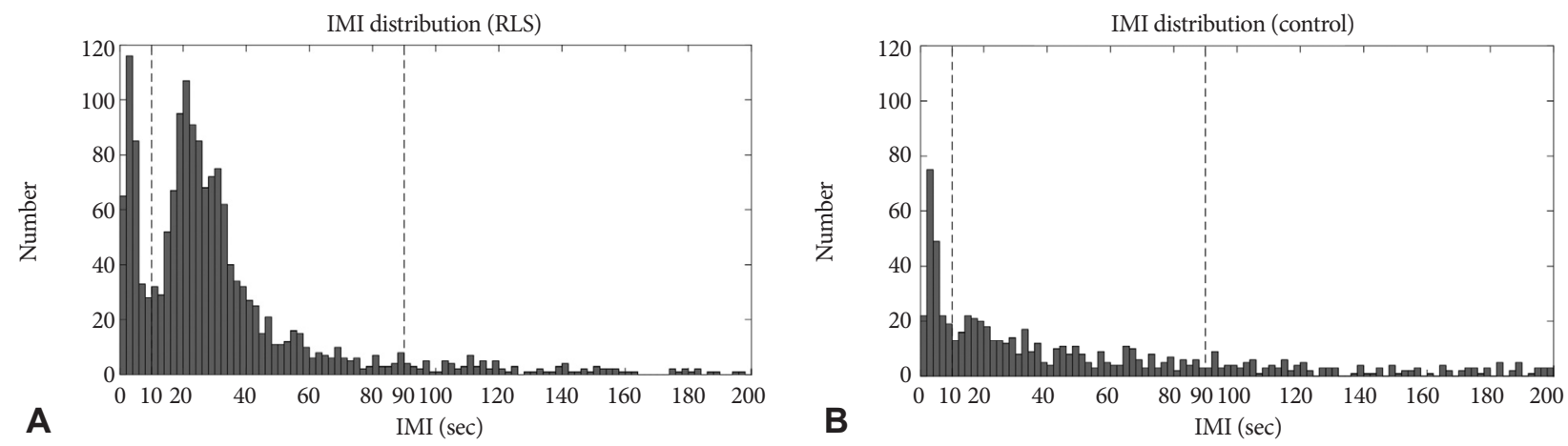

Figure 2. Distribution of IMI in RLS patients (A) and in controls (B). Dashed vertical lines depict boundary IMI for classification of leg movements (IMI=10 s and $90 \mathrm{~s}$ ). IMI: inter-movement interval, RLS: restless legs syndrome.

Table 2. Comparison between LM indices in the patient group and the control group

\begin{tabular}{lccc}
\hline \multicolumn{1}{c}{ LM indices } & RLS $(\mathrm{n}=7)$ & Control $(\mathrm{n}=9)$ & $p$-value \\
\hline PLMS index $(/ \mathrm{h})$ & $28.01(5.91-55.20)$ & $4.04(1.51-8.70)$ & $<0.01^{*}$ \\
SILMS index $(/ \mathrm{h})$ & $9.29(5.05-15.68)$ & $4.49(1.13-7.99)$ & 0.14 \\
ILMS index $(/ \mathrm{h})$ & $8.86(6.73-13.33)$ & $8.55(6.13-11.28)$ & 0.60 \\
\hline
\end{tabular}

Data are presented as median (interquartile range). Mann-Whitney $\mathrm{U}$ test. ${ }^{*} p$-values $<0.05$. LM: leg movements, RLS: restless legs syndrome, PLMS: periodic leg movements in sleep, SILMS: shortinterval leg movements in sleep, ILMS: isolated leg movements in sleep

as $2-5 \mathrm{~s}$ before the onset of LM. The difference between heart rate change associated with ILMS and PLMS does not reach statistical significance except one time point (13 s before the onset). In the control group, heart rate change related to SILMS, ILMS, and PLMS does not show significant difference.

\section{Comparison of average heart rate change between the groups}

RLS patients presented higher heart rate change associated with SILMS $(p=0.011)$ and ILMS $(p=0.026)$ than controls, while heart rate change associated with PLMS were not different between patients and controls $(p=1.000)$.

\section{Discussion}

In order to evaluate clinical significance of NPLM in patients with RLS, PLMS was defined according to WASM crite- 

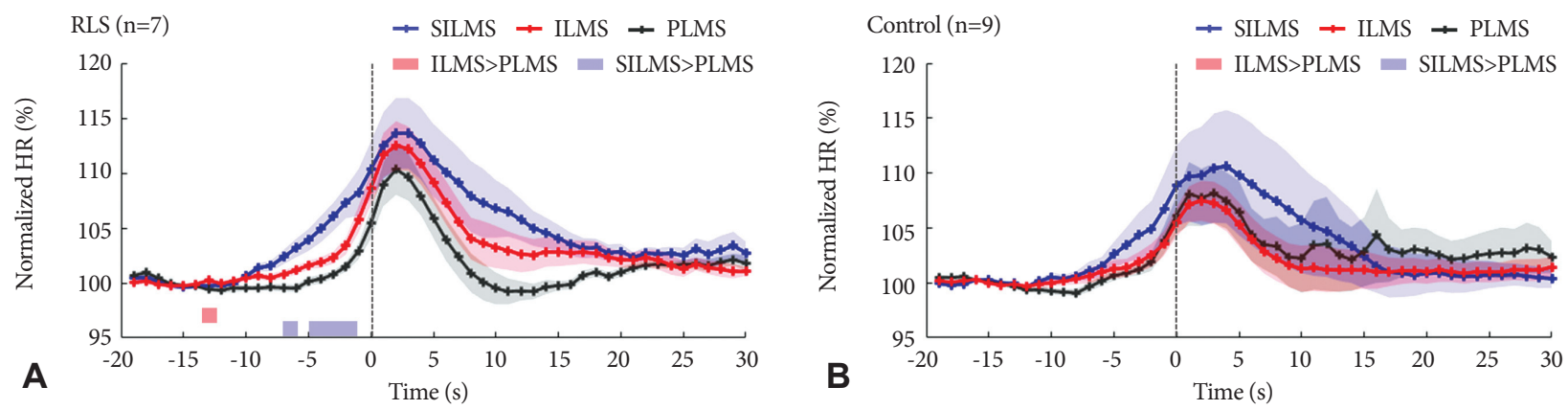

Figure 3. Changes in HR associated with LM in the RLS patient group (A) and in the control group (B). The shaded area depicts the mean \pm the standard error over three different LM types.Vertical dashed line indicates the onset of LM. The bold color lines show statistical significance of difference at each time point between SILMS and PLMS, and difference between ILMS and PLMS (Wilcoxon signed-rank test, $p<0.005)$. HR: heart rate, LM: leg movements, RLS: restless legs syndrome, SILMS: short-interval leg movements in sleep, PLMS: periodic leg movements in sleep, ILMS: Isolated leg movements in sleep.

Table 3. Average heart rate associated with different leg types in the patient group and control group

\begin{tabular}{lcc}
\hline $\begin{array}{c}\text { Average heart } \\
\text { rate (\%) }\end{array}$ & RLS (n=7) & Control (n=9) \\
\hline SILMS & $103.21(102.28-107.05)$ & $100.14(99.92-104.05)$ \\
ILMS & $101.84(101.80-105.01)$ & $100.58(99.71-104.16)$ \\
PLMS & $101.15(100.60-102.79)$ & $100.26(99.68-104.70)$ \\
$\begin{array}{c}p \text {-value } \\
\quad \text { SILMS vs. } \\
\quad \text { PLMS } \\
\text { ILMS vs. } \\
\quad \text { PLMS }\end{array}$ & $0.02^{*}$ & 0.82 \\
$\quad \begin{array}{c}\text { SILMS vs. } \\
\text { ILMS }\end{array}$ & $0.03^{*}$ & 0.82 \\
$\begin{array}{c}\text { Kruskal-Wallis } \\
p \text {-value }\end{array}$ & 0.11 & 0.57 \\
\hline
\end{tabular}

Data are presented as median (interquartile range). Wilcoxon signed-rank test. ${ }^{*} p$-values $<0.05$. RLS: restless legs syndrome, SILMS: short-interval leg movements in sleep, ILMS: isolated leg movements in sleep, PLMS: periodic leg movements in sleep

ria instead of AASM criteria in the present study. This is due to the fact that recent data driven study showed the IMI of all LM have a bi-modal distribution with a low point between the modes at an IMI of about 10 s. ${ }^{15,16}$

We analyzed heart rate change associated with SILMS, ILMS, and PLMS for both groups. Patients with RLS have higher PLMS index than controls. The second peak representing PLMS in the distribution of IMI was distinct only in the patient group. On the other hand, no significant difference in frequencies of SILMS and ILMS indexes between patients and controls are found in this study. Heart rate change according to LM types was significantly different in RLS but not in controls.

Heart rate change associated with PLMS was not different between RLS patients and controls in this study. This result is not consistent with the study of Manconi et al., in which the amplitude of PLMS-related heart rate changes was higher in patient than in controls. ${ }^{17}$ This inconsistency might originate from a large variability of PLMS in the patients with RLS analyzed in this study.

In the time course, ILMS-related heart rate change is not significantly different from PLMS in patients with RLS although average heart rate change related to ILMS is higher than PLMS in patients. The discrepancy in results might be due to limited number of patients included in the study. Heart rate elevation related to SILMS is significantly longer than heart rate elevation related to PLMS in patients with RLS. However, heart rate does not show difference by LM type in healthy controls. This finding reveals that SILMS is accompanied with greater cardiac activation than PLMS in patients with RLS. This finding raises the hypothesis that SILMS has bigger contribution to cardiovascular risk of patients with RLS. If this would be true, treatment to reduce SILMS would also be needed to prevent cardiovascular risk of patients with RLS.

NPLM presents different pharmacological response from PLMS. PLMS and sensory symptoms of RLS are improved with low dose of D3 selective dopamine agonists such as pramipexole. From this pharmacological relationship, PLMS and RLS seem to share same pathogenesis of the dopaminergic deficit. ${ }^{18}$ However, ILMS does not respond to pramipexole treatment. ${ }^{19}$ The occurrence rate of SILMS decreases up to $30 \%$ after acute treatment with low-dose clonazepam, while PLMS is not modified by GABAergic medications. ${ }^{20}$ Therefore, the finding in this study that SILMS is accompanied with greater cardiac activation than PLMS in patients with RLS generates interest on the impact of GABAergic pathway in patients with RLS, especially with cardiovascular risk. Further investigation of change of NPLM and heart rate change related to NPLM after pharmacological treatment of RLS is required.

A limitation of this study is that it was based on a small 
number of subjects. Another limitation is that our study did not consider relationships between SILMS, ILMS, and EEG arousals. Pennestri et al. revealed that PLMS accompanied with microarousals are associated with significant increase in heart rate and blood pressure than PLMS without microarousals. ${ }^{21}$ Further studies of the relationship between NPLM and arousals are needed.

In conclusion, SILMS may have closely associated with higher cardiac activation of RLS than PLMS. Therefore, SILMS might be an important treatment target for patients with RLS to reduce long-term cardiovascular risk. Long-term prospective studies are needed to evaluate the relationship between NPLM and cardiovascular disease in patients with RLS.

\section{Supplementary Materials}

The online-only Data Supplement is available with this article at https:// doi.org/10.13078/jsm.18015.

\section{Acknowledgments}

This work was supported by a National Research Foundation of Korea (NRF) grant funded by the Korean government (MSIP) (2017R1A2B 2012280) and the Brain Research Program through an NRF grant funded by the Ministry of Science, ICT \& Future Planning (2017M3C7A1029485).

\section{REFERENCES}

1. Ferri R, Fulda S, Allen RP, et al. World Association of Sleep Medicine (WASM) 2016 standards for recording and scoring leg movements in polysomnograms developed by a joint task force from the International and the European Restless Legs Syndrome Study Groups (IRLSSG and EURLSSG). Sleep Med 2016;26:86-95.

2. Montplaisir J, Boucher S, Poirier G, Lavigne G, Lapierre O, Lespérance P. Clinical, polysomnographic, and genetic characteristics of restless legs syndrome: a study of 133 patients diagnosed with new standard criteria. Mov Disord 1997;12:61-65.

3. Shin JW, Koo YS, Lee BU, et al. Prevalence and characteristics of periodic limb movements during sleep in Korean adult patients with restless legs syndrome. J Clin Sleep Med 2016;12:1089-1097.

4. Sasai-Sakuma T, Stefani A, Sato M, Högl B, Inoue Y. Ethnic differences in periodic limb movements during sleep in patients with restless legs syndrome: a preliminary cross-sectional study of Austrian and Japanese clinical population. Sleep Biol Rhythms 2018;16:345-349.

5. Ferri R, Zucconi M, Rundo F, Spruyt K, Manconi M, Ferini-Strambi L. Heart rate and spectral EEG changes accompanying periodic and nonperiodic leg movements during sleep. Clin Neurophysiol 2007;118:438448.

6. Walters AS, Rye DB. Review of the relationship of restless legs syn- drome and periodic limb movements in sleep to hypertension, heart disease, and stroke. Sleep 2009;32:589-597.

7. Ferini-Strambi L, Walters AS, Sica D. The relationship among restless legs syndrome (Willis-Ekbom disease), hypertension, cardiovascular disease, and cerebrovascular disease. J Neurol 2014;261:1051-1068.

8. Byun JI, Jung KY, Lee GT, Kim CK, Kim BM. Spontaneous low-frequency cerebral hemodynamics oscillations in restless legs syndrome with periodic limb movements during sleep: a near-infrared spectroscopy study. J Clin Neurol 2016;12:107-114.

9. Ferri R, Rundo F, Silvani A, et al. Short-interval leg movements during sleep entail greater cardiac activation than periodic leg movements during sleep in restless legs syndrome patients. J Sleep Res 2017;26:602-605.

10. Guggisberg AG, Hess CW, Mathis J. The significance of the sympathetic nervous system in the pathophysiology of periodic leg movements in sleep. Sleep 2007;30:755-766.

11. Choi JW, Jeong MH, Her SJ, et al. Abnormal sleep delta rhythm and interregional phase synchrony in patients with restless legs syndrome and their reversal by dopamine agonist treatment. J Clin Neurol 2017;13: 340-350.

12. Berry RB, Brooks R, Gamaldo CE, et al. The AASM manual for the scoring of sleep and associated events: rules, terminology and technical specifcations, version 2.5. Darien: American Academy of Sleep Medicine, 2018.

13. Moore H 4th, Leary E, Lee SY, et al. Design and validation of a periodic leg movement detector. PLoS One 2014;9:e114565.

14. Ferri R, Zucconi M, Manconi M, Plazzi G, Bruni O, Ferini-Strambi L. New approaches to the study of periodic leg movements during sleep in restless legs syndrome. Sleep 2006;29:759-769.

15. Ferri R, Rundo F, Zucconi M, et al. Putting the periodicity back into the periodic leg movement index: an alternative data-driven algorithm for the computation of this index during sleep and wakefulness. Sleep Med 2015;16:1229-1235.

16. Ferri R, Rundo F, Zucconi M, et al. Diagnostic accuracy of the standard and alternative periodic leg movement during sleep indices for restless legs syndrome. Sleep Med 2016;22:97-99.

17. Manconi M, Ferri R, Zucconi M, et al. Effects of acute dopamine-agonist treatment in restless legs syndrome on heart rate variability during sleep. Sleep Med 2011;12:47-55.

18. Saletu M, Anderer P, Saletu-Zyhlarz G, Hauer C, Saletu B. Acute placebo-controlled sleep laboratory studies and clinical follow-up with pramipexole in restless legs syndrome. Eur Arch Psychiatry Clin Neurosci 2002;252:185-194.

19. Manconi M, Ferri R, Feroah TR, Zucconi M, Ferini-Strambi L. Defining the boundaries of the response of sleep leg movements to a single dose of dopamine agonist. Sleep 2008;31:1229-1237.

20. Ferri R, Rundo F, Silvani A, et al. Sequence analysis of leg movements during sleep with different intervals $(<10,10-90$ and $>90 \mathrm{~s})$ in restless legs syndrome. J Sleep Res 2017;26:436-443.

21. Pennestri MH, Montplaisir J, Colombo R, Lavigne G, Lanfranchi PA. Nocturnal blood pressure changes in patients with restless legs syndrome. Neurology 2007;68:1213-1218. 
Supplementary Table 1. Individual indices of SILMS, ILMS, and PLMS

\begin{tabular}{|c|c|c|c|c|c|c|}
\hline \multirow{2}{*}{ Subjects } & \multicolumn{3}{|c|}{ Control $(n=9)$} & \multicolumn{3}{|c|}{$\operatorname{RLS}(\mathrm{n}=7)$} \\
\hline & SILMS index $(/ \mathrm{h})$ & ILMS index (/h) & PLMS index (/h) & SILMS index $(/ \mathrm{h})$ & ILMS index $(/ \mathrm{h})$ & PLMS index (/h) \\
\hline Sub 1 & 1.23 & 2.93 & 0.46 & 5.60 & 8.86 & 5.91 \\
\hline Sub 2 & 3.67 & 9.26 & 2.23 & 3.37 & 6.73 & 55.20 \\
\hline Sub 3 & 10.65 & 8.55 & 9.20 & 9.80 & 7.50 & 39.49 \\
\hline Sub 4 & 1.03 & 7.05 & 8.21 & 15.68 & 10.56 & 17.70 \\
\hline Sub 5 & 4.49 & 6.40 & 2.59 & 5.05 & 4.42 & 4.84 \\
\hline Sub 6 & 5.52 & 9.50 & 5.13 & 68.72 & 18.77 & 88.02 \\
\hline Sub 7 & 6.06 & 13.07 & 4.04 & 9.29 & 13.33 & 28.01 \\
\hline Sub 8 & 0.78 & 5.85 & 0.78 & & & \\
\hline Sub 9 & 9.91 & 15.14 & 14.59 & & & \\
\hline
\end{tabular}

Data are presented as median. SILMS: short-interval leg movements in sleep, ILMS: isolated leg movements in sleep, PLMS: periodic leg movements in sleep, RLS: restless legs syndrome 